\title{
Oct4 Protein and Gene Expression Analysis in the Differentiation of Spermatogonia Stem Cells Into Functional Mature Neurons by Immunohistochemistry, Immunocytochemistry, and Bioinformatics Analysis
}

Hossein Azizi ( $\square$ h.azizi@ausmt.ac.ir)

Amol University of Special Modern Technologies

Danial Hashemi Karoii

Amol University of Special Modern Technologies

Thomas Skutella

Heidelberg University

Research Article

Keywords: Spermatogonia Stem Cells, Oct4, Neuron, Reprogramming, Pluripotency

Posted Date: January 17th, 2022

DOI: https://doi.org/10.21203/rs.3.rs-1231019/v1

License: (1) (i) This work is licensed under a Creative Commons Attribution 4.0 International License.

Read Full License 


\section{Abstract}

Spermatogonia Stem Cells (SSCs) are potential candidates for reprogramming, therapeutic neuronal repair, and regeneration. Recent studies have revealed that differentiated cells can be reverted to a pluripotent state by overexpressing a set of pluripotent transcription factors. Oct4 (encoded by pou $5 \mathrm{f} 1$ ), a POU transcription factor family member, is essential to the potential that controls pluripotency, and it is widely expressed in pluripotent stem cells, although it decreased or suppressed after differentiation. In this investigated research, we examined the Oct4 Expression in vivo and in-vitro of SSCs, Embryonic Stem Cell-like (ES-like), Embryonic Bodies (EBs), and Neurons by Immunocytochemistry (ICC), Immunohistochemistry (IMH), and Fluidigm Real-Time polymerase chain reaction. In addition, We use some databases like STRING to predict protein-protein interaction and enrichment analysis. We evaluated the expression of Oct4 in this process, and we observed that it is expressed in SSCs, ES-like, and EBs during the differentiation of spermatogonia stem cells into adult neurons. We show that by adding RA to EBs, the Expression of Oct4 is reduced and is not expressed in the neuron cells. We observed that the Expression of Oct4 is linked and interacts with the differentiation of spermatogonia stem cells into neuron cells, and it has been shown to be biological functional like stem cell maintenance and somatic cell reprogramming. Our findings can help us better understand the process of differentiation of spermatogonia stem cells into neurons, and it can be effective in finding new and more efficient treatments for neurogenesis and repair of neurons. Moreover, this study may comprehend the new Oct4 reprogramming method for clinical and therapeutic use.

\section{Introduction}

Due to the limited capability of the nervous system in neuronal repairers, stem cells therapy that promotes neurogenesis and repair the wounded regions are proposed (Kahroba, Ramezani et al. 2021). For this reason, the capacity of stem cells to develop into diverse lineages, pluripotent stem cell therapy is a viable therapeutic method for the treatment of neurodegenerative disease (Bojnordi, Azizi et al. 2017, Maher 2019). Several studies have shown that embryonic stem cell-derived neural can help therapy in neural animal models (Strnadel, Carromeu et al. 2018). In addition, because of immunological and ethical constraints, embryonic stem cells (ESCs) are not employed in therapeutic methods. As a result, it is appropriate to seek a changeable safe cell source that may overcome these ethical and technological constraints and enable autologous transplantation, reducing the possibility of immunological rejection transplantation (Little, Ketteler et al. 2019). According to a new study, Embryonic Stem Cell-like (ES-like) derived from the spermatogonial stem cells (SSCs) exhibit similar molecular and cellular profiles to ESCs and contained pluripotent stem cell properties. Some studies show that they can give rise to all three germ layers in-vitro and in-vivo (Hall 2018, Shafa, Yang et al. 2018).

The initial experiments demonstrated that the production of brain cell types from mouse ESCs relied on the formation of embryoid bodies (EBs) intermediates. ESC-derived EBs are comparable to teratocarcinoma stem cell-derived embryoid entities (Katayama, Morii et al. 2018). The EBs resemble the anterior prestreak stage embryo in this stage, with the epiblast-like core able to produce derivatives of all 
three primary germ layers. The embryoid body core maintains the ESC marker Oct4 and expresses the primitive ectoderm marker FGF5 (Bojnordi, Azizi et al. 2017, Yang, Wu et al. 2017).

Combinations of pluripotent genes (Oct4, Sox2, Nanog, and cMyc) for reprogramming somatic or adult stem cells or inducing pluripotent cell-specific factors have been reported to produce highly effective results in pluripotent cell reprogramming (Zhang, Skamagki et al. 2017). Oct4 (encoded by Pou5f1), a POU-family transcription factor, is a key pluripotency transcription factor and it is required for ES cells self-renewal and conversion of differentiated cells to induced pluripotent stem cells (Li 2017). As a result, it is widely understood that Oct4 expression levels determine the balance of differentiation and dedifferentiation. Maintaining Oct4 Expression within a specific range seems essential for stem cell renewal, with any rise or reduction triggering three germ layers (Echigoya, Koyama et al. 2020, Zalc, Sinha et al. 2021).

Pluripotent stem cells (PSCs) were produced using various methods, including ESCs collected from the inner cell mass of an embryo (Azizi, Asgari et al. 2019). They were also obtained through the forced expression of pluripotency genes in somatic cells, resulting in induced pluripotent stem cells (iPSCs); SSCs are one of the promising methods for a more natural and ethically unproblematic establishment of PSCs, particularly for therapeutic approaches in human medicine (Conrad, Azizi et al. 2016).

Spermatogonial stem cells (SSCs) are found in the testis in modest numbers, but they can be isolated and grown in vitro. While they are unipotent stem cells under environmental control, particular culture conditions outside the niche, and the absence of any exogenous pluripotency genes, they may convert to ESC-like cells at different intervals following the start of the culture of Spermatogonial stem cells (Azizi, Conrad et al. 2016, Azizi, Hamidabadi et al. 2019).

SSCs are well recognized to be unipotent stem cells that express both pluripotency and germ cell markers. Under germ cell culture conditions, SCCs can spontaneously convert into pluripotent embryonic stem (ES)-like cells (Bojnordi, Azizi et al. 2017). These pluripotent ES-like cells can then differentiate into a wide range of cells lineages. Ko et al. performed the pluripotency induction in 5-7 weeks Oct-4-GFPpositive adolescent SSCs (Azizi, Asgari et al. 2019). The author reported that the initial amount of plated SSCs and the duration of the Oct4-positive cell culture period without splitting affect differentiation induction. They carefully selected heterogonous Oct4-GFP-positive SSCs and revealed a link between a specific number of SSCs and a culture time of 2-4 weeks for pluripotency induction. In another study, Ko et al. (Kaushik and Bhartiya 2018) performed the pluripotency induction in 5-week- to 7-month-old Oct-4 GFP positive adult SSCs and detailed the induction's dependence on the initial quantity of plated SSCs and the duration of culture period of Oct-4-positive cells without splitting. However, in the subsequently published methodology for converting SSCs into pluripotent stem cells, this group primarily used SSCs from mice at postnatal day 35. (5 weeks old) (Akamatsu, DeVeale et al. 2009, Yamashiro, Sasaki et al. 2018).

In the context of past findings demonstrating the pluripotency of ES-like cells derived from the spermatogonial stem cells, the purpose of this research was to evaluate the differentiation capacity and 
changes in Oct- 4 gene expression patterns during in vitro differentiation of spermatogonial stem cells into neurons cells by adding Retinoic acid (RA) in the mouse embryonic bodies.

\section{Materials And Methods}

\section{Testicular cell isolation:}

Amol University of Special Modern Technologies, Amol, Iran, approved the protocols for animal care and surgical intervention of adult mice in this study. Pasteur Institute (Iran) provided male mice (7 weeks old, C57BL/ 6 mice). Phosphate-buffered saline with $2 \%$ BSA and 0.1 percent Triton X100 was used to collect mouse testis. In Dulbecco's modified Eagle's medium, the testis seminiferous tubules were minced into minute pieces (DMEM, Invitrogen, USA). Enzymatic digestion solution containing Dispase $(0.5 \mathrm{mg} / \mathrm{ml})$ (Sigma Aldrich, USA), collagenase IV $(0.5 \mathrm{mg} / \mathrm{ml})$ (Sigma Aldrich, USA), and DNase $(0.5 \mathrm{mg} / \mathrm{ml})$ (Sigma Aldrich, USA) in HBSS buffer with Ca2+ and Mg2+ (PAA, USA) was used to collect testicular cells at $37^{\circ} \mathrm{C}$ for 10 minutes. The cell solution was then rinsed with DMEM/F12 (Invitrogen, USA), passed through a $70 \mathrm{~m}$ mesh filter, and centrifuged at $1500 \mathrm{rpm}$ for 10 minutes. The isolated testicular cells were maintained at 37 degrees Celsius in 5\% $\mathrm{CO} 2$ in the air. Every three days, the culture media was replaced (Azizi, Asgari et al. 2019).

\section{Generation and culture of ES-Like cells from testis:}

As we previously stated, ES-like cells were generated from SSCs (Azizi, Asgari et al. 2019). Oct4 promoterreporter GFP transgenic mice from the C57BL/ 6 adult mouse strains were cultured in a mouse spermatogonia stem cells culture medium. About 6 weeks after culture initiation, ES-like cells with a high level of Oct4-GFP were generated and subcultured in an embryonic stem cells medium for growth (Azizi, Conrad et al. 2016).

\section{Formation of embryoid bodies from ES-like:}

In our study, 2-3 ES-like cells were grown in 10-cm2 bacterial-grade petri plates with low attachment. For 3-4 days, the EBs were formed spontaneously in suspension culture in a sterile bacterial petri dish containing a DMEM with 15\% fetal bovine serum (FBS), 1\% NEAA solution, 1\% L-glutamine, 1\% Pen-Strep, and $0.1 \%$-mercaptoethanol (Bojnordi, Azizi et al. 2017).

\section{Differentiation of neurons from ES-Like cells:}

Three stages of neuronal differentiation of ES-like cells were induced. As previously discussed, stage 1 was related to EB development (Bojnordi, Azizi et al. 2017). In stage 2, EBs were grown in suspension in low-attachment 10-cm2 bacterial-grade petri plates with 1\% L-glutamine, DMEM-F12, , 1\% N2, 1\% NEAA, $10 \mathrm{ng} / \mathrm{ml} \mathrm{bFGF}$, and $500 \mathrm{nM}$ retinoic acid (RA) to generate neural progenitor cells. During the second stage, the FBS concentration was lowered to $10 \%$ (days $1-2$ ), $5 \%$ (days $3-4$ ), and $3 \%$ (days 5-6). Following that, about 10 days after the start of suspension culture, the EBs were plated (which were coated with poly-L-ornithine) on 6- or 24-well tissue culture plates with neurobasal medium, $1 \%$ N2, 5\% 
FBS, $1 \%$ NEAA, $1 \% \mathrm{~L}$ glutamine, and 2\% B27 for an additional 10 days to allow the development of the neuronal progenitor (Azizi, Skutella et al. 2017, Azizi, Hamidabadi et al. 2019).

\section{Patch-Clamp Recording in neural cells cultures:}

Type I and II cells were fixed as a pellet in PIPES with paraformaldehyde/ glutaraldehyde for electron microscopy. The fixative was changed after 10 minutes. Pellets were then post-fixed for 40 minutes in Os04/potassium hexacyanoferrate, washed in uranyl acetate buffer and sodium maleate buffer $(\mathrm{pH} 6.0)$, and block-stained with uranyl acetate. Following dehydration with increasing ethanol concentrations (5 min each, $4 \times 20 \mathrm{~min}$ ), the tissue was Epon-embedded ( $60^{\circ} \mathrm{C}$ polymerizations, $36 \mathrm{~h}$ ). A Zeiss EM10 was used to examine ultrathin slices (50 nm) (Azizi, Skutella et al. 2017, Bojnordi, Azizi et al. 2017).

\section{Fluidigm Dynamic Arrays for Gene Expression Analysis:}

Quantity of the Oct4 gene expression (Mm03053917_g1) in SSCs, ES-like, EBs, Sertoli cells, and Mouse Embryonic Fibroblasts (MEF, for control) was assayed by Fluidigm dynamic array chips. Glyceraldehyde3-phosphate dehydrogenase (Mm99999915_g1) was utilized as a housekeeping gene for normalization. All cells were selected with a micromanipulator, lysed with a lysis buffer solution containing $1.3 \mu \mathrm{lTE}$ buffer, $9 \mu \mathrm{l}$ RT-PreAmp Master Mix, $0.2 \mu \mathrm{l}$ R.T./Taq Superscript III (Invitrogen, USA), $2.5 \mu \mathrm{l}$ 0.2× assay pool, and $5.0 \mu \mathrm{l}$ Cells Direct $2 \times$ Reaction Mix (Invitrogen, USA). The targeted transcripts were quantified using TaqMan real-time PCR on the Biomark Real-Time quantitative PCR system (Fluidigm-PCR)(Azizi, Conrad et al. 2016, Conrad, Azizi et al. 2016).

\section{Immunocytochemical and immunohistofluorescence staining:}

The testicular cell was fixed with $4 \%$ paraformaldehyde and then permeably with $0.1 \%$ Triton/PBS. The testicular cell was blocked with $1 \%$ BSA/PBS and incubation with primary antibody vimentin. Then, We used secondary antibodies specific for incubation fluorochrome species, and the labeled cells were nuclear-counterstained treatment with $0.2 \mu \mathrm{g} / \mathrm{ml}$ of 4', 6-diamidino-2-phenylindole (DAPI) dye. The Labeled positive testicular cells were studied under confocal microscopy Zeiss LSM 700 (Germany), and images were used a Zeiss LSM-TPMT camera (Germany).

In IMH staining, cells were washed with PBS and fixed in 4\%paraformaldehyde. Dehydrated tissues were located in Paraplast Plus and chop with a microtome device at $10 \mu \mathrm{m}$ thickness. Testis tissues section were mounted on Superfrost Plus slides and kept at $25^{\circ} \mathrm{C}$ until used. In the process of IMH staining, samples were washed with xylene and gradually replaced with water in ethanol. For tissues, antigens retrieval was performed by heat-induced epitope retrieval at $94^{\circ} \mathrm{C}$ for 18 minutes. The non-specific binding site of tissue samples was blocked with $10 \%$ serum and $0.3 \%$ Triton in PBS. As explained above, the experiment of immunofluorescence staining for these samples was continued.

\section{Search strategy and data preparation for protein-protein interactions (PPI) network analysis:}


Gene databases (NCBI: https://www.ncbi.nlm.nih.gov/ gene/ and KEGG: https://www.genome.jp/ Kegg/genes/) were searched for gene-related datasets. (Spermatogonia stem cell), (ES-like cell), (Embryonic bodies), (Neurons), and "Mus musculus" [porgn:_txid10090] were the keyword phrases. The gene expression profile was then saved in an excel file. In this section, $\mathrm{P}<0.04$ was used to select gene interactions and clusters.

\section{PPI network analysis:}

STRING (Szklarczyk, Gable et al. 2019) (version 11.5) online tool was applied to predict protein-protein biological and functional interactions (https://string-db.org/ ). The gene-related with a significant role in Oct4 (Pou5f1) were uploaded in the STRING online tool. The master regulator of Pou5f1, gene coexpression, and signaling pathways were highlighted, To identify. The highlighted genes were imported to Cytoscape (Doncheva, Morris et al. 2018) (version 3.9.0) for further analysis and protein-protein interactions network visualization.

\section{Gene set enrichment analysis:}

To confirm the biological roles of the genes involved in the PPI network of first co-expression nodes with Oct4 (Pou5f1), we have performed functional gene enrichment analysis using the STRING enrichment analysis in the Cytoscape software.

\section{Statistical analysis}

The tests were performed at least three times. The average gene expression in each group was assessed, and the experimental data were analyzed using a one-way analysis of variance (ANOVA) followed by Tukey's post-hoc testing.

\section{Ethical statement:}

In this study, all animal experiments were approved (Approval ID: Ir.ausmt.rec.1398.03.07) to the local and international guidelines for the use of experimental animals and were approved by the ethics committee of Amol University of Special Modern Technologies and all sections of this report adhere to the ARRIVE Guidelines for reporting animal research.

\section{Results}

\section{Oct4's expression in seminiferous tubules by immunohistochemical:}

In this experiment, we first analyzed Oct4's Expression in the section of the mouse seminiferous tubules by immunohistochemical (IMH). Oct4 was the high expression in spermatogonia stem cells located in the basal compartment of seminiferous tubules (Fig.1 stage $A$ and $B$ ).

SSCs isolated and Oct4's Expression by immunocytochemical: 
Then, SSCs are isolated after enzyme digestion, and the cells isolated are cultured in the presence of growth factors supporting SSC culture. Characterization of isolated SSCs was performed as described in our previous study. We examined the Expression of Oct4 in SSCs populations by immunocytochemical (ICC). Images obtained from the confocal scanning UV-laser microscope in ICC analysis demonstrated high Oct4 expression SSCs (Fig.1 stage C).

\section{ES-Like characteristics and formation of EBs:}

In culture, ES-like colonies, which are compact colonies with sharp edges, formed(Fig.2 stage A). Their phenotypic resembled that of ES colonies. After 3-4 days of culture on low attachment bacterial-grade petri plates, EB structures were developed, which can be regarded as a distinct feature of pluripotent ESlike cells (Fig.2 stage B and C).

\section{Oct4 Gene expression analyses:}

Fluidigm real-time RT-PCR results showed high Expression of Oct4 mRNA in SSCs and low Expression in Sertoli cells. In addition, the Oct4 Expression of ES-like cells and embryonic bodies is higher than SSCs (Fig. 3).

\section{Electrophysiological dates of neurons:}

We investigated the functioning of neuron-derived ES-like cells. The patch-clamp recording was used to determine the functional status of induced neurons at the conclusion of the differentiation process (Fig. 4 stage A). We examined resting membrane and action potentials in 15 neuron-like cells. Following the injection of depolarizing and hyperpolarizing currents, the membrane voltage was measured using the current-clamp mode. The recorded resting membrane potential varied between 20 and $30 \mathrm{mV}$. Before injecting currents, the voltage was set at $65 \mathrm{mV}$. We observed a single spike in the induced neurons but no repeated spike firing following the current injection. Spikes used to have a maximum amplitude of roughly $40 \mathrm{mV}$ (Fig. 4 stage B1). This study demonstrated that in vitro stimulation of ES-like cells results in the formation of neurons, which mostly expressed neural markers and demonstrated preliminary electrical properties of neurons. The firing of repeated spikes stopped after pretreatment with TTX (1 M), indicating the presence of sodium currents in neurons after the induction process (Fig. 4 stage B2).

\section{Differentiation of neurons from ES-Like Cells:}

After being exposed to in vitro neural inducers (Retinoic acid, RA), ES-like cells demonstrated the ability to differentiate into neural cells. Neuron-like cells were seen 6 days after the initiation of neural induction, and their numbers rapidly increased. Regardless of the fact that non-treated ES-like cells spontaneously differentiated into neurons, just a few neuron cells were seen in this group. The results of real-time PCR demonstrated the Expression of Nestin and PAX6 (neuroprogenitor markers) in differentiated cells during stage 2 culture, indicating a considerable rise in gene expression level in undifferentiated ES-like cells during the first stage (Fig. 5). With this procedure, significant levels of expression of the neuronal genes 
MAP2, Tuj1, neuN, Tau were found in the cells after 10 days of treatment, demonstrating the ability of neural precursors to differentiate into mature neurons.

However, the MAP2 gene was shown to be expressed at a low level in neural progenitors (Fig. 5 stage B). Immunostaining demonstrated neurogenic differentiation for mature neuron markers. Tuj1 and Tau1 were among the mature neural markers expressed by multipolar neurons. In noninduced ES-like cells, the expression of these proteins was undetectable (Fig. 5). The molecular results were supported by immunocytochemistry labeling of the brain markers Tuj1 and Tau1. We looked for the expression of mature neuron markers at the conclusion of the neural induction process for ES-like cells. Tuj1 and Tau1 antibodies were used to label cells grown on coverslips (Fig. 5 stage $C$ and D).

\section{PPI visualization of Oct4:}

Protein-protein interactions network was visualized gen using STRING (v.11.5) database. It demonstrated that there was a close relationship between interaction and regulated Oct4 (Pou5f1) in SSCs, ES-like, EBs, and neurons. We observe a high interaction (co-expression) between Myc, Cdh1, Stat3, Ctnnb1, SOX2, NANOG, GATA4, and Ezh1 with Oct4 (Pou5f1) in SSCs (Fig. 6 stage A). In addition, there was an association between Pml, H3f3a, SOX2, NANOG, Ncor1, SOX17, Dnmt3a, and Ctnnb1 with Oct4 (Pou5f1) in ES-like (Fig. 6 stage B). Furthermore, there was an association between SOX2, NANOG, Ctcf, Hdac1, Trim28, and Ctnnb1 with Oct4 (Pou5f1) in EBs (Fig. 6 stage C). Moreover, there was an association between H3f3a, Ehmt2, Ep300, Ctnnb1, and Esrrb with Oct4 (Pou5f1) in neurons (Fig. 6 stage D). There is a strong correlation between the highlighted genes, as is clear (Fig. 6). All this PPI process was done by Cytoscape software.

\section{Functional enrichments in PPI network:}

Enrichment analysis was carried out to recognize enriched biological processes and molecular functions associated with Oct4 (Pou5f1) and its first co-expression proteins (Fig. 7). We selected some biological processes based on the purpose of our experiment, including positive regulation of neural precursor cell proliferation, positive regulation of stem cell proliferation, regulation of cell division, DNA binding, Ubiquitin protein ligase binding, and miRNA binding (Fig. 7 A-D).

\section{Statistical Analysis:}

The statistical analyses were carried out using the SPSS 16.0 program and the independent-sample Student's t-test. $P$ values were less than 0.05 , indicating statistical significance.

\section{Discussion}

The idea that the fundamental pluripotency factors Oct4 play a key role in maintaining cell pluripotency and lineage specification has caught the interest of stem cell researchers since it bridges the mechanistic transition from a pluripotent stem cell to a lineage-specific cell type. We observed Oct4 was the high Expression in SSCs located in the basal compartment of seminiferous tubules, and when we 
differentiation SSCs into ES-like cells, the Expression of Oct4 increases. During ES-like cells differentiation into neurons, first ES-like cells differentiate into EBs, and by adding RA, they differentiate to neurons. In this process, we added RA to EBs, and we observed that the expression of oct4 was decreased (Fig. 8). Some studies show that Oct4 Expression is confined to the germ cell lineage after gastrulation (Zhang, Bell et al. 2019), and germ cell nuclear factor, an orphan nuclear receptor, suppresses Oct4 Expression in the early neuroectoderm by GCNF (Lei, Tian et al. 2020, Ding, Lan et al. 2021). We suggested that by reducing Oct4 Expression, neurons differentiate faster and better.

STRING database shows that Oct4 (pou5f1) is associated with pluripotent genes in the SSCs, ES-like, and EBs stages and performs functions such as positive regulation of neural precursor cell proliferation, positive regulation of stem cell proliferation, regulation of cell division, DNA binding, Ubiquitin protein ligase binding, and miRNA binding. We suggest that by differentiating neuron cells from SSCs, the function of Oct4 in neuronal and neuronal stem cells is suppressed. STRING and Cytoscape analysis presented five genes, Oct4, GATA4, Ctnnb1, NANOG, and SOX2, as the hub genes involved in these processes.

We demonstrated an in vitro differentiation process for the generation of functional neurons from mouse SSCs. In this process, neuroprogenitor cells were generated from ES-like cells, which are capable of generating functioning neurons, and then differentiated further. The electrophysiological evaluation data indicated that the generated neurons functioned. Cell therapy successfully treats neurodegenerative diseases and improves central nervous system functional recovery (Azizi, Conrad et al. 2016). Pluripotent stem cells are the most excellent choice for this purpose due to their utility as a source of cell transplantation. Many clinical constraints associated with embryonic stem cells, on the other hand, suggest an alternative cell source for therapeutic methods. The potential of ESC to differentiate into neural progenitors and functioning neurons has been widely described. However, due to immunological and ethical constraints, human ESCs do not have therapeutic uses (Nogales, Prat et al. 2018, Azizi, Asgari et al. 2019).

As a result, it is critical to establish an alternative safe cell population capable of overcoming ethical and technological limitations. The results of this study suggested that SSCs, like ESCs, had the potential for brain differentiation. After being exposed to neural inducers, ES-like cell colonies demonstrated neural differentiation, including neuroprogenitors and mature neurons. The neuroprogenitor cells were generated after culturing EBs in a medium supplemented with RA, N2, and FGF. Nestin and PAX6 markers were revealed on neuroprogenitor cells, which are involved in neurogenesis throughout embryo development. Furthermore, following incubation in a neurobasal medium enriched with NEAA, B27, and N2, neuroprogenitor cells matured and developed. Our discovery backs up earlier findings on the generation of neuroprogenitor cells from ESCs.

Furthermore, we observed that under the presence of NCAA, B27, and N2, neuroprogenitors could be increased and expanded. This data is consistent with the findings of other researchers who reported the formation of neural progenitors from ESCs in the presence of N2B27 media supplemented with growth 
factors. After being cultivated in a neurobasal media containing NEAA, B27, and N2, neuroprogenitor cells grew and differentiated into neurons. According to immunocytochemistry labeling, the neuronal markers Tuj1 and Tau1 were expressed in differentiated cells at the end of the differentiation phase. Similar data investigating the differentiation potential of ESC development into mature neurons indicated the expression of these mature neural markers. The electrophysiological observations of neuroprogenitor cells cultured in the specified culture after 10 days demonstrated the functional properties of neurons.

This research supports previous findings that ESCs can generate mature neurons (Bojnordi, Azizi et al. 2017). The expression of pluripotency, neuroprogenitor, and mature neuron markers was widely studied throughout the neural development of ES-like cells. Although ES-like cells were shown to express several pluripotency markers (Nanog, C-myc, Nestin), Neuro D markers were related to neuroprogenitor cells. Neuroprogenitor cells were differentiated into a neuron phenotype based on morphology and high expression levels of mature neuronal markers Map2 and -tubulin in the final stage (stage 3). After 10-12 days of treatment with inducers, significant levels of neuronal gene expression were identified in the cultures, suggesting the differential potential of neural progenitors to mature neurons. The electrical characteristics of differentiated neurons were studied using patch-clamp recordings. The current study confirmed the electrical properties of neuronal-like cells produced from ES-like cells obtained from the testis. Although additional research is required to assess the fully functional phenotype of ES-like cells produced from neurons, our results imply that the neural inducers used in this study enhanced ES-like cell differentiation to the neural lineage. However, inducing more mature neurons through various protocols based on a co-culture system with supporting cells via the preparation of essential developmental factors in the maturation of the functioning neuron is more important (Villodre, Felipe et al. 2019).

Previously researchers demonstrated that replacing cell populations such as ESCs or ESC-derived neuroprogenitor cells with adult neurons improved experimental models of neurodegenerative diseases (Simmet, Zakhartchenko et al. 2018). Despite improvements, teratoma formation rates following undifferentiated ESC transplantation are much higher than after transplantation of highly differentiated cells, neuroprogenitors, or completely differentiated neurons derived from ESCs. In vitro neural differentiation of ESCs prior to transplantation outperforms the engraftment of undifferentiated stem or neuroprogenitor cells to solve this challenge. In this context, we hoped to promote neuron development in ES-like cells derived from the testis and treated with ESC-specific neural procedures. Furthermore, adding survival reagents to culture media, such as NEAA, B27, N2, and bFGF, increases both viability and eventual differentiation of ESC-derived neurons.

We previously demonstrated the glial differentiation capability of SSCs derived from mouse testis that revealed a functional profile after transplantation into multiple sclerosis demyelination models (Conrad, Azizi et al. 2016, Bojnordi, Azizi et al. 2017). Our study reveals that ES-like cells derived from the testis may develop into mature neurons and exhibit functional characteristics of neural progenitors and mature neurons. It seems that testicular ES-like cells can be used to replace a variety of neuroglial cells. This breakthrough expands the scope of neurological research and medical applications. Furthermore, the approach used to differentiate normal neurons from ESCs also applies to testicular ES-like cells. In vitro, 
they can develop into fully functional adult neurons. However, further research must validate these cells' ability to function in injured host brain regions in vivo.

\section{Conclusion}

In summary, our study evaluated the Oct4 expression pattern during testicular cells, ES-like, EBs, and neurons. Furthermore, we analyzed its association with other genes and their connections in the PPI network using STRING: PubMed query and Cytoscape software. Our findings can help us better understand the process of differentiation of spermatogonia stem cells into functional mature neurons, and it can be effective in finding new and more efficient treatments for neurogenesis and repair of nerves. Also, comprehend the new Oct4 reprogramming method for clinical and therapeutic use. Finally, future studies should pay more attention to Oct4 connected networks to improve our current understanding of Oct4 performance.

\section{Declarations}

\section{Acknowledgments:}

This research was supported by Amol University of Special Modern Technologies (AUSMT) and the University of Heidelberg, Institute for Anatomy and Cell Biology III, Department of Neuroanatomy, Germany.

Funding: none.

\section{Authors' Contributions:}

H.A: Carried out and designed the experiment and edited the manuscript; D.H.K: Assembly of data, data analysis, design PPI network and enrichment analysis, wrote and edited the manuscript; T.S; Provided critical feedback, data analysis, and edited the final manuscript.

\section{Conflict of Interests:}

The authors declare that there is no conflict of interest regarding the publication of this study.

\section{References}

1. Akamatsu, W., B. DeVeale, H. Okano, A. J. Cooney and D. van der Kooy (2009). "Suppression of Oct4 by germ cell nuclear factor restricts pluripotency and promotes neural stem cell development in the early neural lineage." Journal of Neuroscience 29(7): 2113-2124.

2. Azizi, H., B. Asgari and T. Skutella (2019). "Pluripotency potential of embryonic stem cell-like cells derived from mouse testis." Cell Journal (Yakhteh) 21(3): 281. 
3. Azizi, H., S. Conrad, U. Hinz, B. Asgari, D. Nanus, H. Peterziel, A. Hajizadeh Moghaddam, H. Baharvand and T. Skutella (2016). "Derivation of pluripotent cells from mouse SSCs seems to be age dependent." Stem cells international 2016.

4. Azizi, H., H. G. Hamidabadi and T. Skutella (2019). "Differential proliferation effects after short-term cultivation of mouse spermatogonial stem cells on different feeder layers." Cell Journal (Yakhteh) 21(2): 186.

5. Azizi, H., T. Skutella and A. Shahverdi (2017). "Generation of mouse spermatogonial stem-cellcolonies in a non-adherent culture." Cell Journal (Yakhteh) 19(2): 238.

6. Bojnordi, M. N., H. Azizi, T. Skutella, M. Movahedin, F. Pourabdolhossein, A. Shojaei and H. G. Hamidabadi (2017). "Differentiation of spermatogonia stem cells into functional mature neurons characterized with differential gene expression." Molecular neurobiology 54(7): 5676-5682.

7. Conrad, S., H. Azizi, M. Hatami, M. Kubista, M. Bonin, J. Hennenlotter, K.-D. Sievert and T. Skutella (2016). "Expression of genes related to germ cell lineage and pluripotency in single cells and colonies of human adult germ stem cells." Stem cells international 2016.

8. Ding, S., X. Lan, Y. Meng, C. Yan, M. Li, X. Li, J. Chen and W. Jiang (2021). "CHD8 safeguards early neuroectoderm differentiation in human ESCs and protects from apoptosis during neurogenesis." Cell death \& disease 12(11): 1-15.

9. Doncheva, N. T., J. H. Morris, J. Gorodkin and L. J. Jensen (2018). "Cytoscape StringApp: network analysis and visualization of proteomics data." Journal of proteome research 18(2): 623-632.

10. Echigoya, K., M. Koyama, L. Negishi, Y. Takizawa, Y. Mizukami, H. Shimabayashi, A. Kuroda and H. Kurumizaka (2020). "Nucleosome binding by the pioneer transcription factor OCT4." Scientific reports 10(1): $1-11$.

11. Hall, B. K. (2018). "Germ layers, the neural crest and emergent organization in development and evolution." genesis 56(6-7): e23103.

12. Kahroba, H., B. Ramezani, H. Maadi, M. R. Sadeghi, H. Jaberie and F. Ramezani (2021). "The role of Nrf2 in neural stem/progenitors cells: From maintaining stemness and self-renewal to promoting differentiation capability and facilitating therapeutic application in neurodegenerative disease." Ageing research reviews 65: 101211.

13. Katayama, S., A. Morii, J. O. Makanga, T. Suzuki, N. Miyata and T. Inazu (2018). "HDAC8 regulates neural differentiation through embryoid body formation in P19 cells." Biochemical and biophysical research communications 498(1): 45-51.

14. Kaushik, A. and D. Bhartiya (2018). "Pluripotent very small embryonic-like stem cells in adult testesan alternate premise to explain testicular germ cell tumors." Stem cell reviews and reports 14(6): 793-800.

15. Lei, I., S. Tian, V. Chen, Y. Zhao and Z. Wang (2020). "SWI/SNF component BAF250a coordinates OCT4 and WNT signaling pathway to control cardiac lineage differentiation." Frontiers in cell and developmental biology 7: 358 . 
16. Li, Y.-Q. (2017). "Networks of transcription factors for Oct4 expression in mice." DNA and cell biology 36(9): 725-736.

17. Little, D., R. Ketteler, P. Gissen and M. J. Devine (2019). "Using stem cell-derived neurons in drug screening for neurological diseases." Neurobiology of aging 78: 130-141.

18. Maher, P. (2019). "The potential of flavonoids for the treatment of neurodegenerative diseases." International journal of molecular sciences 20(12): 3056.

19. Nogales, F. F., J. Prat, M. Schuldt, N. Cruz-Viruel, B. Kaur, E. D'Angelo, X. Matias-Guiu, A. Vidal, W. G. McCluggage and J. W. Oosterhuis (2018). "Germ cell tumour growth patterns originating from clear cell carcinomas of the ovary and endometrium: a comparative immunohistochemical study favouring their origin from somatic stem cells." Histopathology 72(4): 634-647.

20. Shafa, M., F. Yang, T. Fellner, M. S. Rao and B. A. Baghbaderani (2018). "Human-induced pluripotent stem cells manufactured using a current good manufacturing practice-compliant process differentiate into clinically relevant cells from three germ layers." Frontiers in medicine 5: 69.

21. Simmet, K., V. Zakhartchenko, J. Philippou-Massier, H. Blum, N. Klymiuk and E. Wolf (2018). "OCT4/POU5F1 is required for NANOG expression in bovine blastocysts." Proceedings of the National Academy of Sciences 115(11): 2770-2775.

22. Strnadel, J., C. Carromeu, C. Bardy, M. Navarro, O. Platoshyn, A. N. Glud, S. Marsala, J. Kafka, A. Miyanohara and T. Kato (2018). "Survival of syngeneic and allogeneic iPSC-derived neural precursors after spinal grafting in minipigs." Science translational medicine 10(440).

23. Szklarczyk, D., A. L. Gable, D. Lyon, A. Junge, S. Wyder, J. Huerta-Cepas, M. Simonovic, N. T. Doncheva, J. H. Morris and P. Bork (2019). "STRING v11: protein-protein association networks with increased coverage, supporting functional discovery in genome-wide experimental datasets." Nucleic acids research 47(D1): D607-D613.

24. Villodre, E. S., K. B. Felipe, M. Z. Oyama, F. H. de Oliveira, P. L. da Costa Lopez, C. Solari, G. Sevlever, A. Guberman and G. Lenz (2019). "Silencing of the transcription factors Oct4, Sox2, Klf4, c-Myc or Nanog has different effect on teratoma growth." Biochemical and biophysical research communications 517(2): 324-329.

25. Yamashiro, C., K. Sasaki, Y. Yabuta, Y. Kojima, T. Nakamura, I. Okamoto, S. Yokobayashi, Y. Murase, Y. Ishikura and K. Shirane (2018). "Generation of human oogonia from induced pluripotent stem cells in vitro." Science 362(6412): 356-360.

26. Yang, J., C. Wu, I. Stefanescu and A. Horowitz (2017). "Analysis of retinoic acid-induced neural differentiation of mouse embryonic stem cells in two and three-dimensional embryoid bodies." JoVE (Journal of Visualized Experiments)(122): e55621.

27. Zalc, A., R. Sinha, G. S. Gulati, D. J. Wesche, P. Daszczuk, T. Swigut, I. L. Weissman and J. Wysocka (2021). "Reactivation of the pluripotency program precedes formation of the cranial neural crest." Science 371(6529).

28. Zhang, C., M. Skamagki, Z. Liu, A. Ananthanarayanan, R. Zhao, H. Li and K. Kim (2017). "Biological significance of the suppression of oxidative phosphorylation in induced pluripotent stem cells." Cell 
reports 21(8): 2058-2065.

29. Zhang, S., E. Bell, H. Zhi, S. Brown, S. A. Imran, V. Azuara and W. Cui (2019). "OCT4 and PAX6 determine the dual function of SOX2 in human ESCs as a key pluripotent or neural factor." Stem cell research \& therapy $10(1): 1-14$.

\section{Figures}
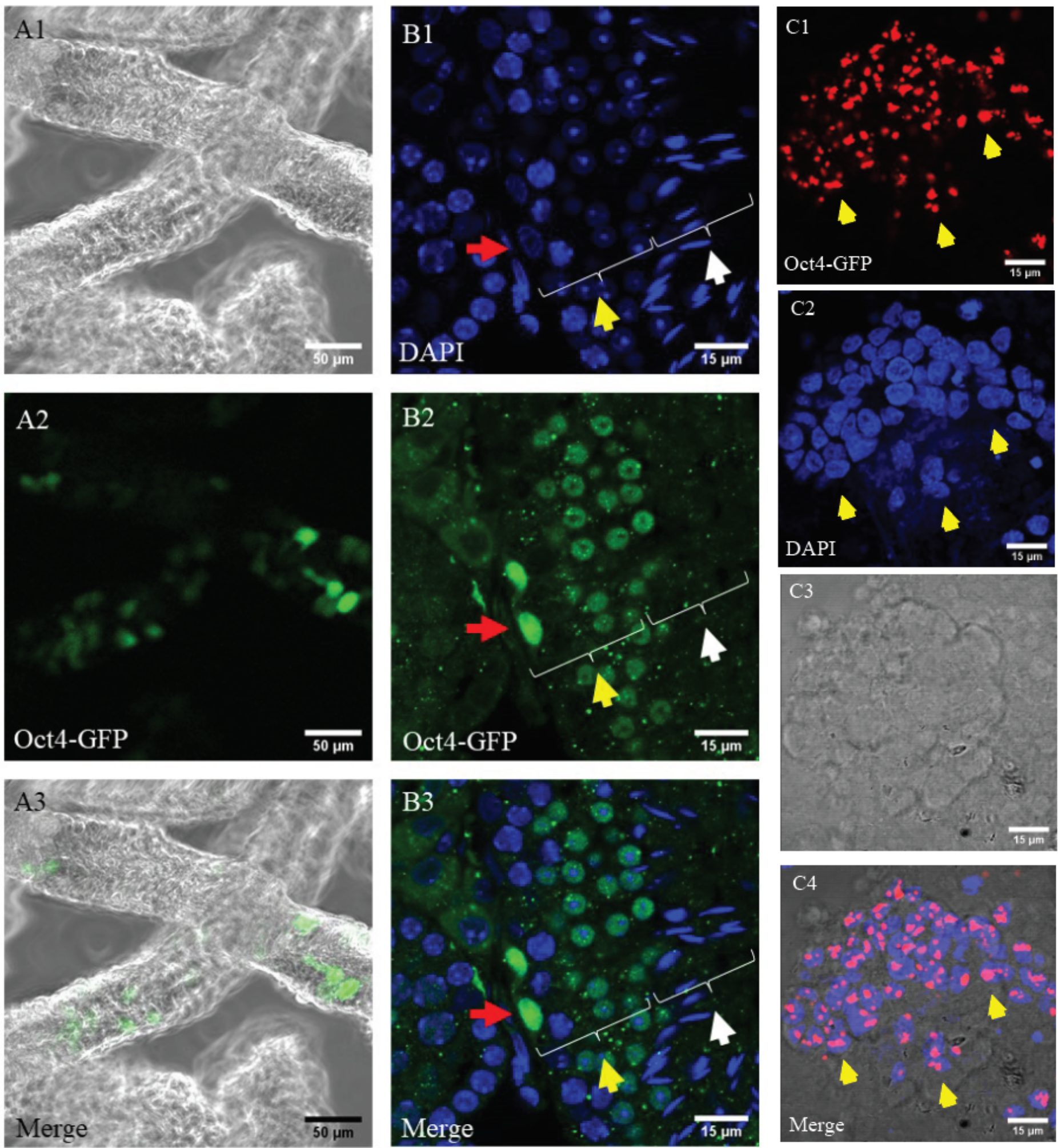


\section{Figure 1}

The intensity and phase-contrast microscopy of Oct4-GFP signals in adult mouse testis and in vitro. $(a, b)$ Oct4 expression in seminiferous tubules (c) SSC cultures from transgenic Oct4-GFP reporter mice. (A1A3) Oct4-GFP positive cells and the intensity of the Oct-GFP signal were higher in testicular tubules of adult mice. (B1-B3) Oct4 was the high Expression in SSCs located in the basal compartment of seminiferous tubules (yellow arrow), although it was not expressed in SSCs cells in the middle and central of seminiferous tubules (white arrow). (C1-C3) Oct4-GFP positive SSCs were present during initial cultures from adult mice. Scale bars: (a) $50 \mu \mathrm{m}$, (b-c) $15 \mu \mathrm{m}$. 

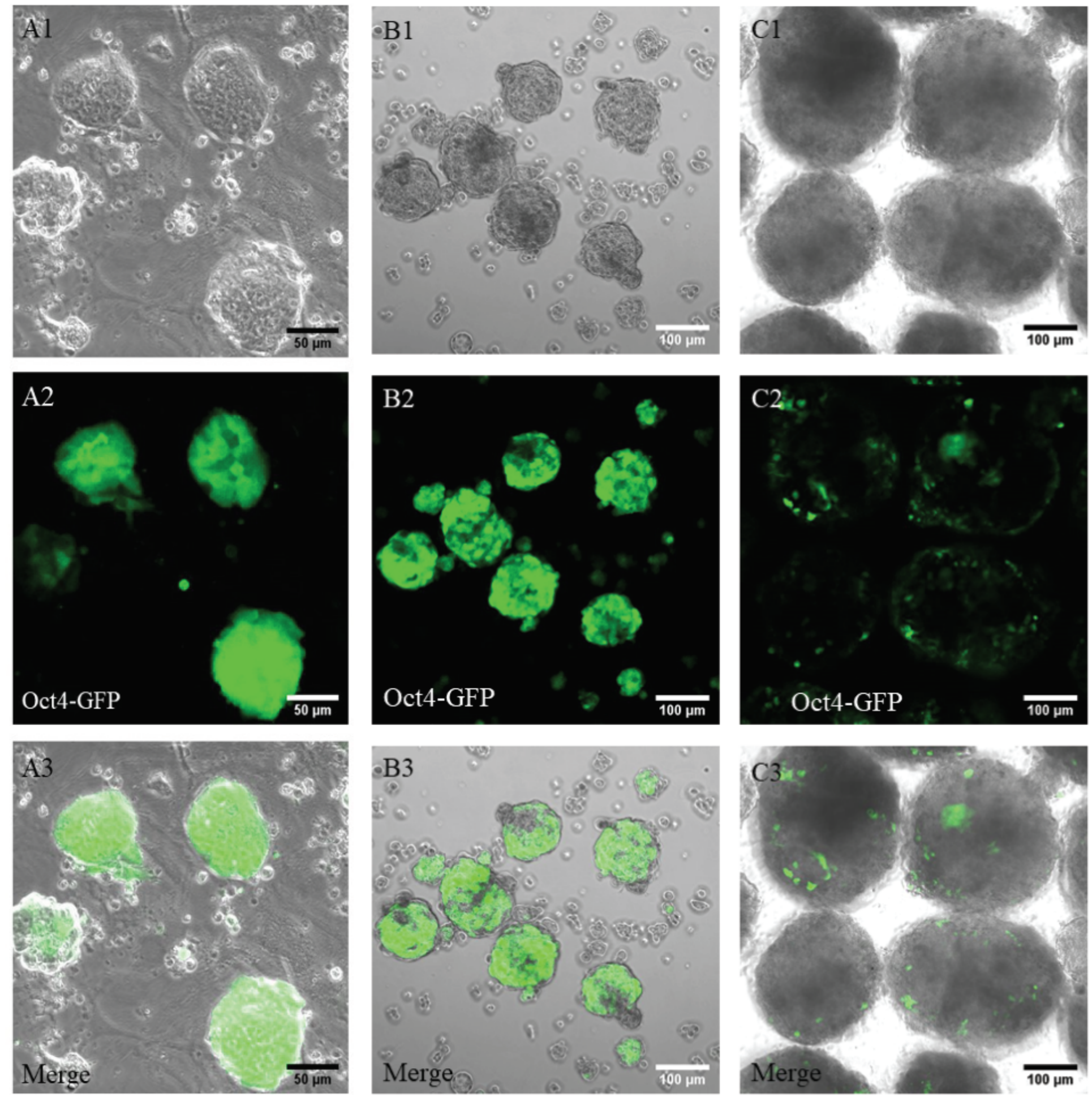

\section{Figure 2}

The intensity and phase-contrast microscopy figure of ES-like cells and EBs after expansion. (a) Immunofluorescence microscopy figure of ES-like cells shows Oct4-GFP. (b) EBs structures were formed in suspension culture after expansion. (c) Treating an EBs by adding RA. At this stage, Oct4-GFP Expression reaches a less low level. Scale bars: (a) $50 \mu \mathrm{m}$ and (b-c) $100 \mu \mathrm{m}$. 


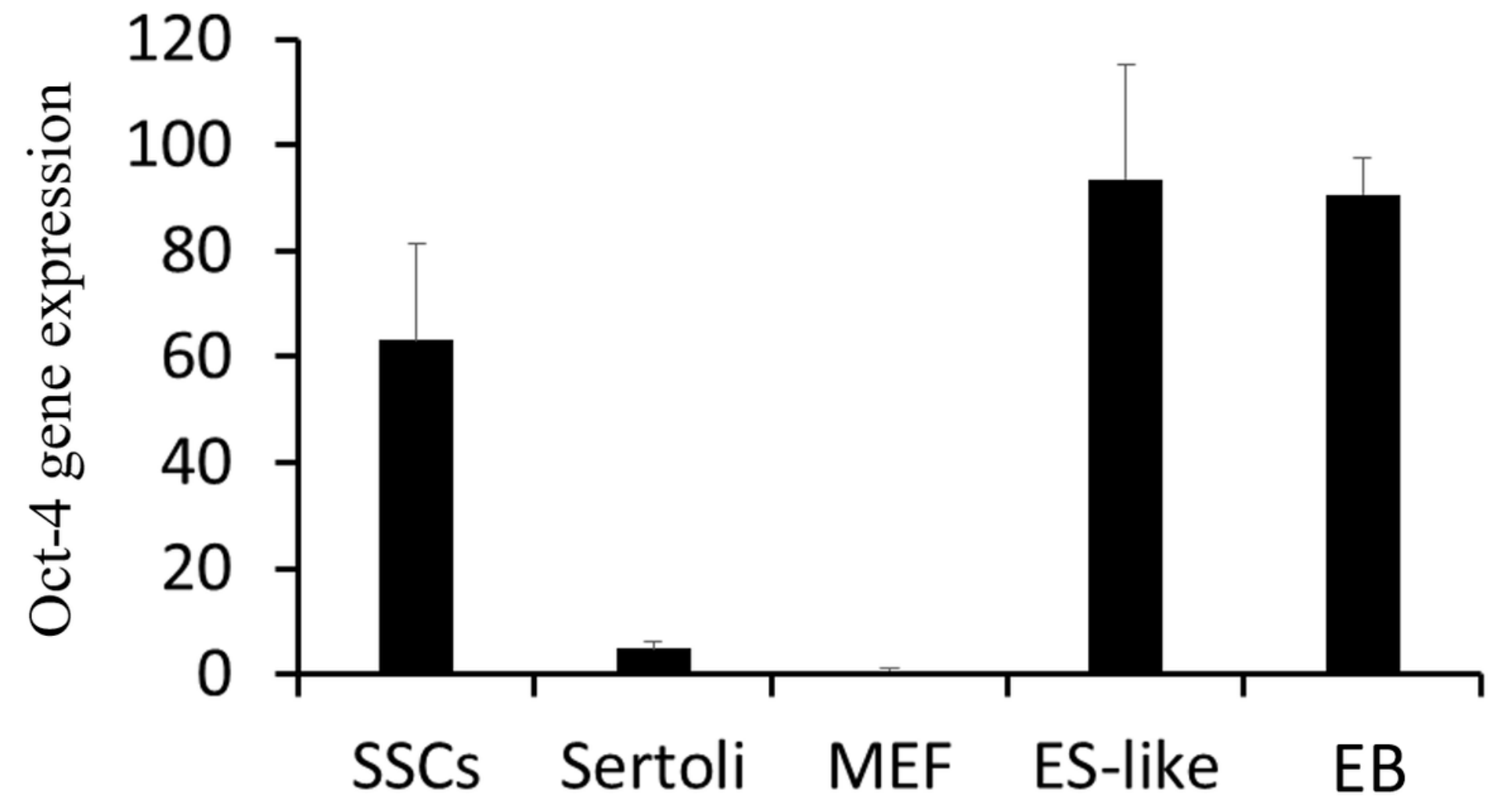

Figure 3

Oct4 mRNA expression of SSCs, Sertoli, ES-like, and EBs. Fluidigm quantitative polymerase chain reaction analysis for Oct4 Expression in SSCs, Sertoli, ES-like cells, EBs, and MEF; Mouse embryonic fibroblasts (for control). High mRNA expressed in undifferentiation spermatogonia, and very high mRNA expressed in ES-like cells and EBs. At least $\mathrm{P}<0.05$ versus other groups. Data presented as mean $\pm \mathrm{SD}$. 

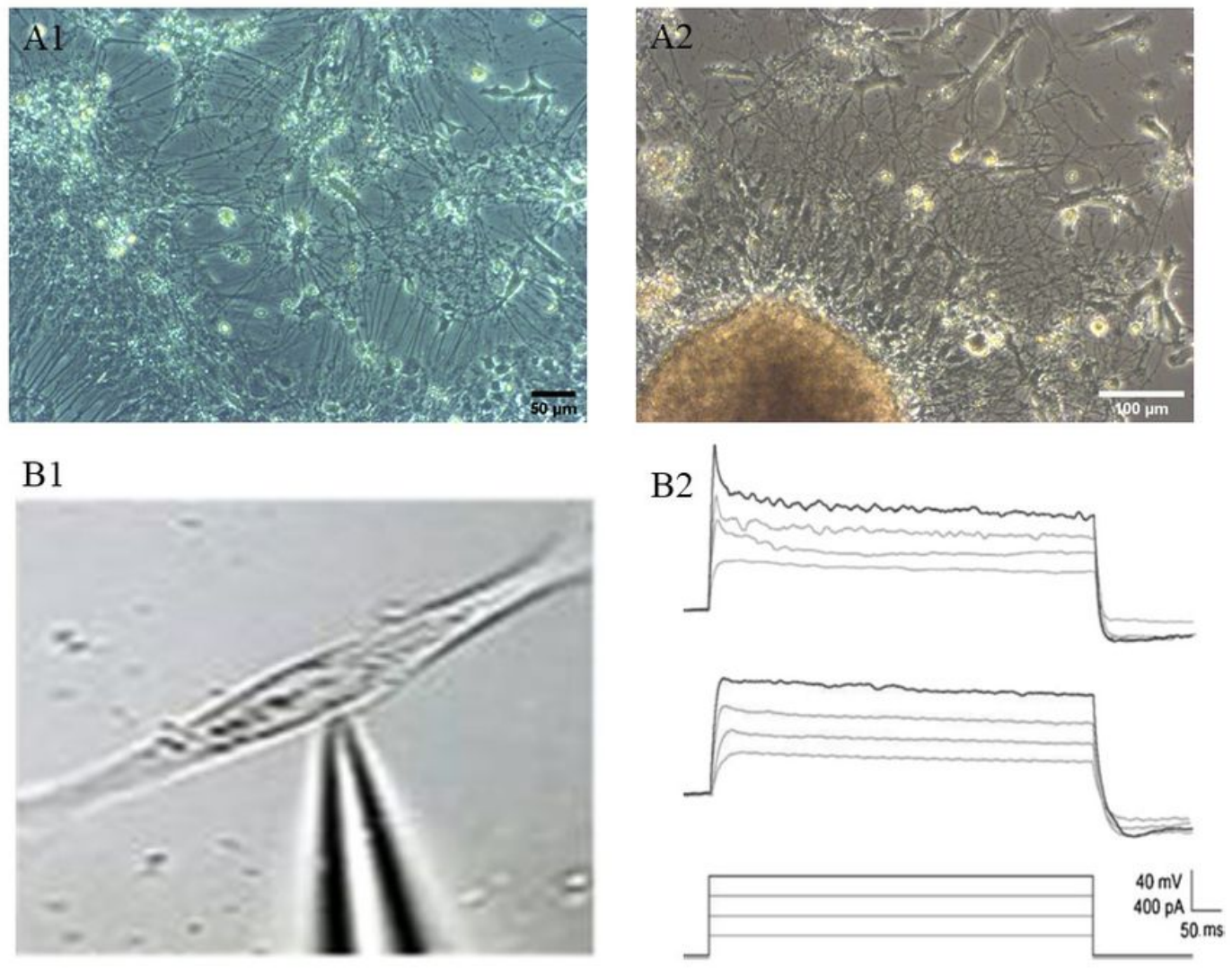

\section{Figure 4}

phase-contrast microscopy and patch clamp recording of neurons. (a) Neuron cells were seen 6 days after the initiation of neural induction, and their numbers rapidly increased. (b) Whole-cell current-clamp recordings of differentiated neurons. Scale bars: (a) $50 \mu \mathrm{m}$, (b) $15 \mu \mathrm{m}$. 

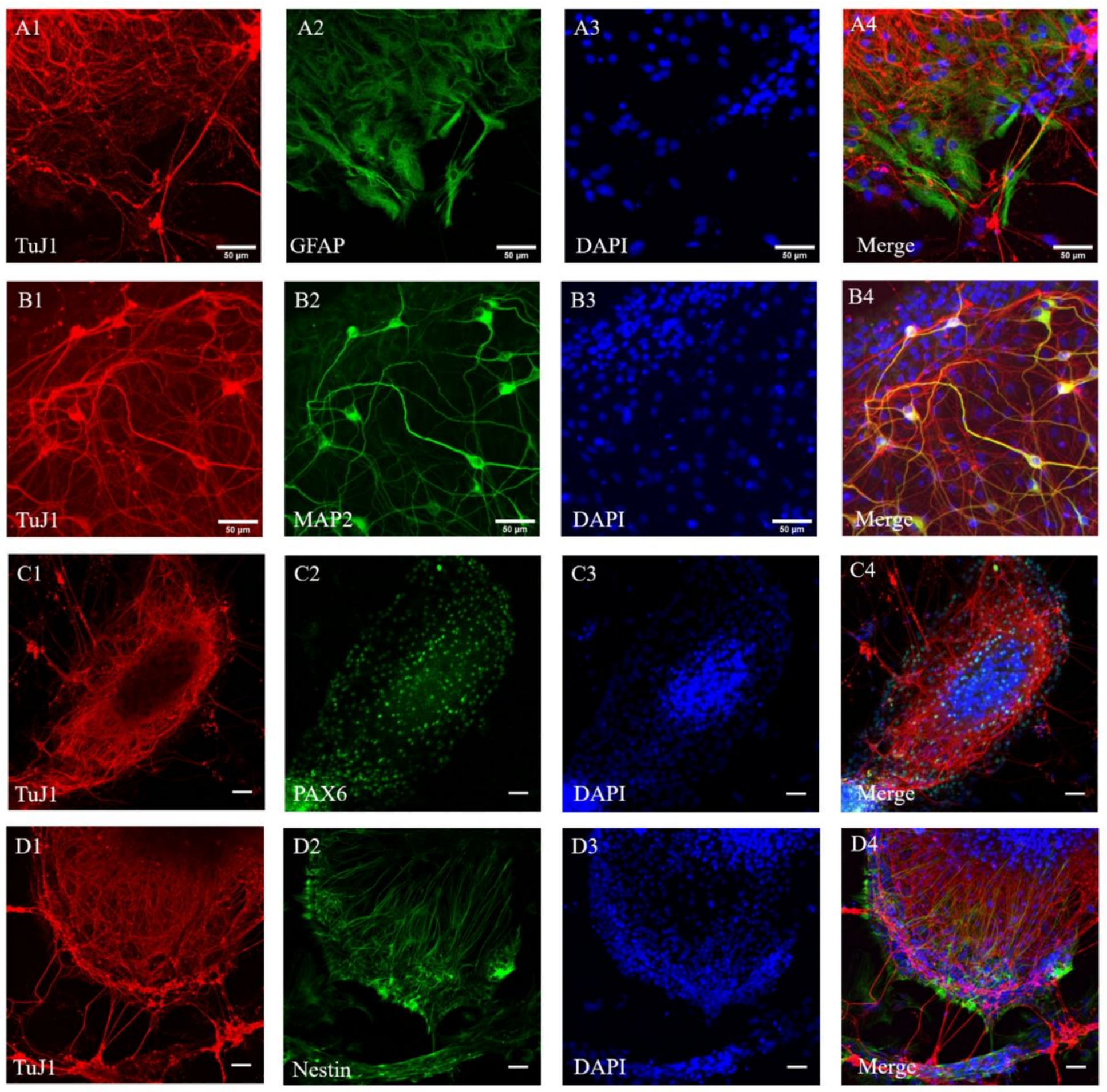

Figure 5

Differentiation of neurons from ES-Like Cells. (A-D) Neurons induced for an additional 10 days at the final stage of the differentiation. Scale bars, $50 \mu \mathrm{m}$. 
A

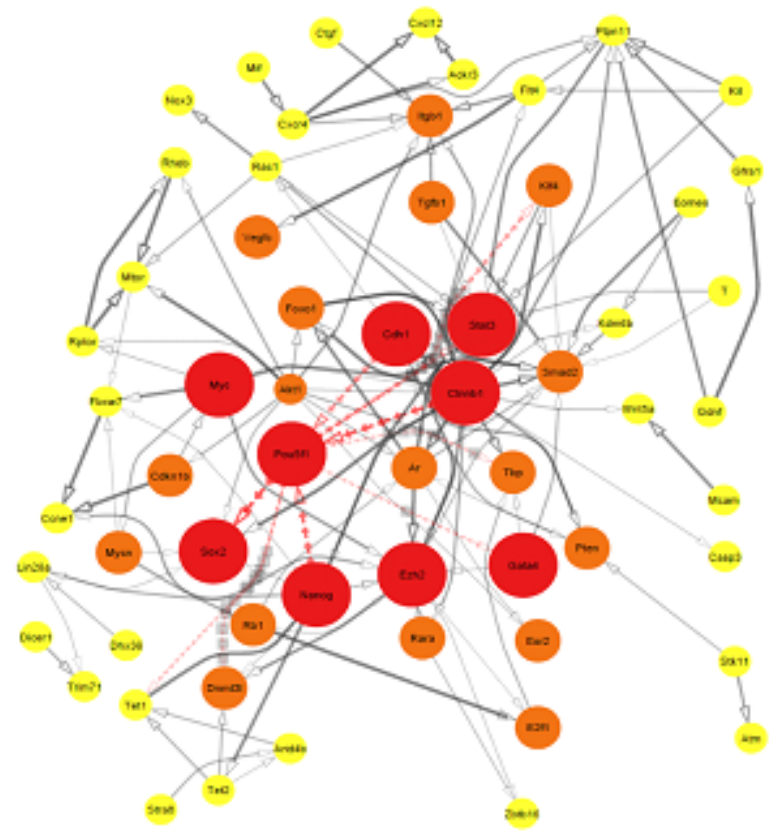

C

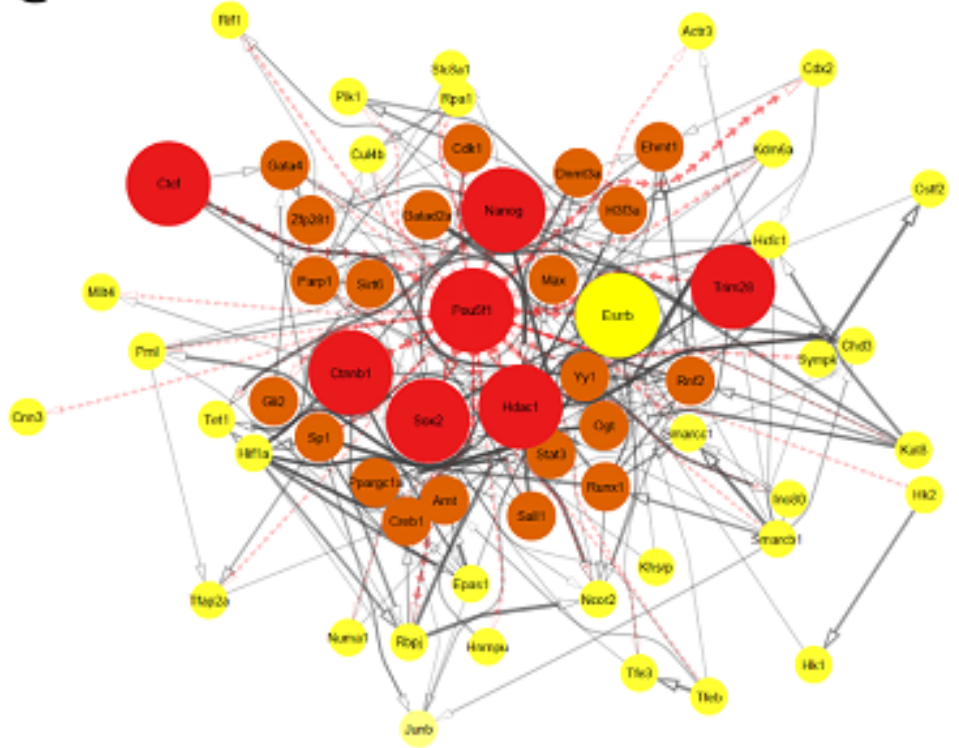

B

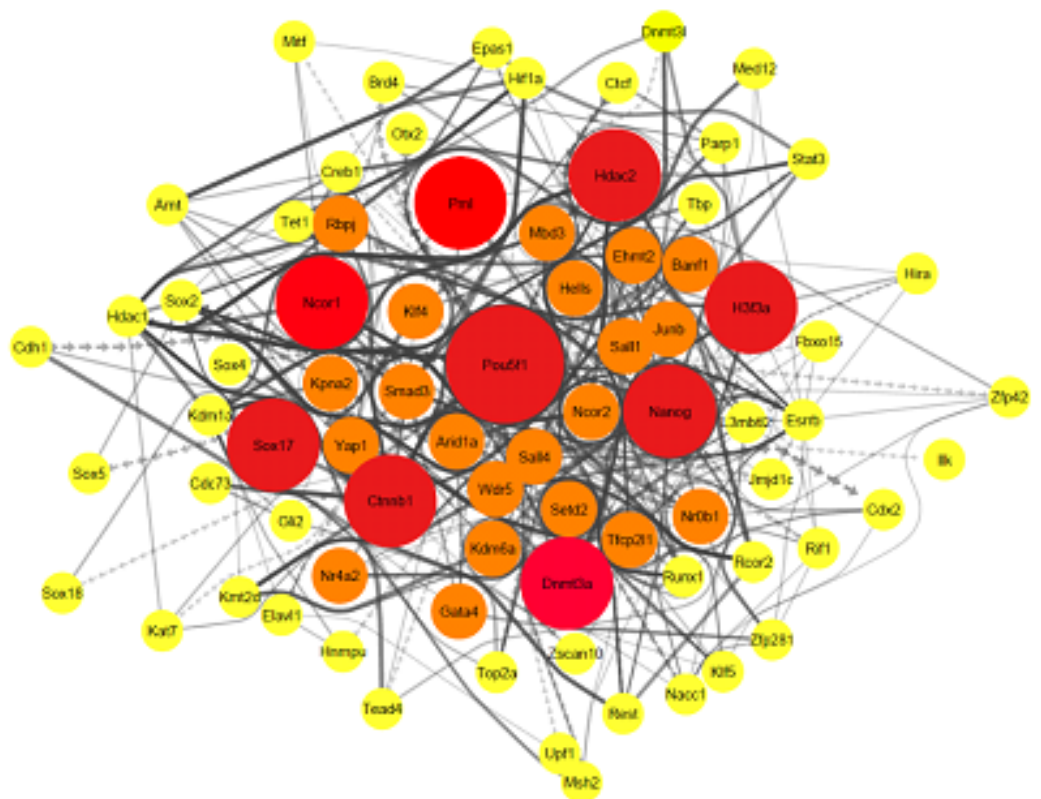

D

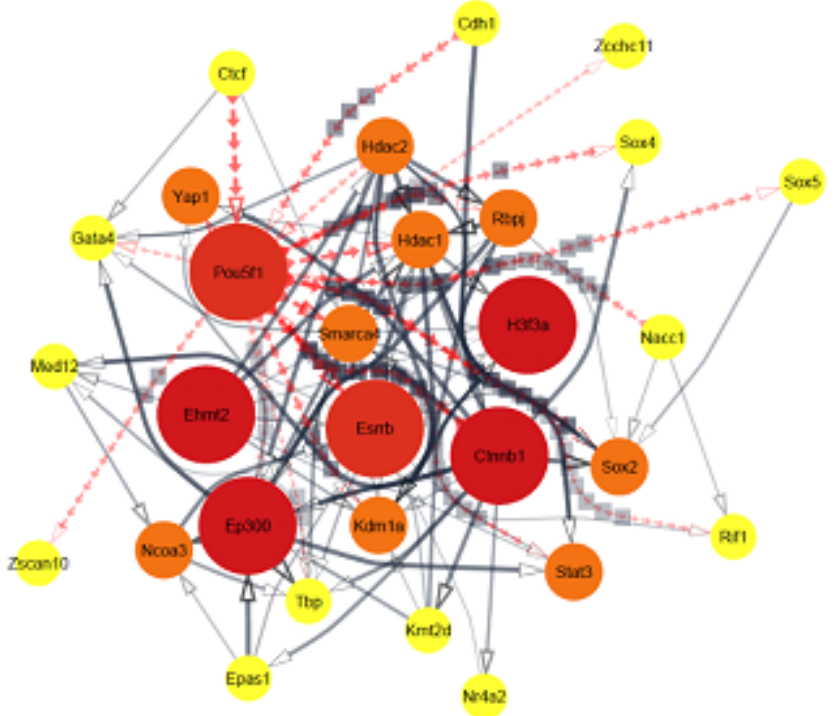

Figure 6

The protein-protein interaction (PPI) Network analysis is involved in spermatogonial stem cell to neural.

The network was constructed by Cytoscape based on the PubMed query from the STRING database. The maximum number of proteins was set as 100 with a confidence score extracted $\mathrm{P}<0.40$. The red node represents high related and co-expression with Pou5f1. (a) related and co-expression genes with Pou5f1 in SSCs. (b) related and co-expression genes with Pou5f1 in ES-like. (c) related and co-expression genes with Pou5f1 in EBs. (d) related and co-expression genes with Pou5f1 in neurons. 


\begin{tabular}{|c|c|c|c|c|}
\hline $\begin{array}{l}\text { GO-fern and } \\
\text { pathways }\end{array}$ & Discerplion & $\begin{array}{l}\text { Count in } \\
\text { network }\end{array}$ & FDR & Color \\
\hline GO:0051302 & Regulation of cell division & 3 of 197 & 0.0303 & \\
\hline GO:1905114 & $\begin{array}{l}\text { Cell suffice receppor signaling } \\
\text { pathway involved in cell-sell }\end{array}$ & 4 of 352 & 0.0141 & \\
\hline GO:0003677 & DNA binding & 15 of 192 & $1.38 \mathrm{e}-08$ & \\
\hline GO:0033044 & $\begin{array}{l}\text { Regulation of chromosome } \\
\text { of gantization }\end{array}$ & 404363 & 0.0154 & \\
\hline GO:0048863 & Stem cell defferentiation & 6 of 174 & $2.60 \mathrm{e}-06$ & \\
\hline MQMU-212436 & Generic Transcription Pathway & 8 of 834 & 0.0120 & \\
\hline GO:0031625 & Ubiquitin protein ligase binding & 4 of 303 & 0.0226 & \\
\hline $\operatorname{mmu} 04550$ & $\begin{array}{l}\text { Signaling pathways regulating } \\
\text { pluripotency of stem cells }\end{array}$ & 9 of 137 & $3.97 \mathrm{e} \cdot 12$ & \\
\hline
\end{tabular}

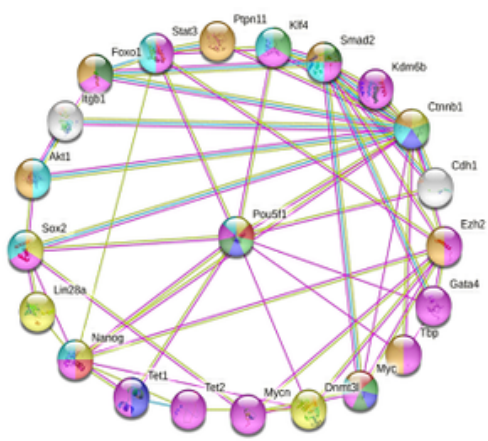

B1

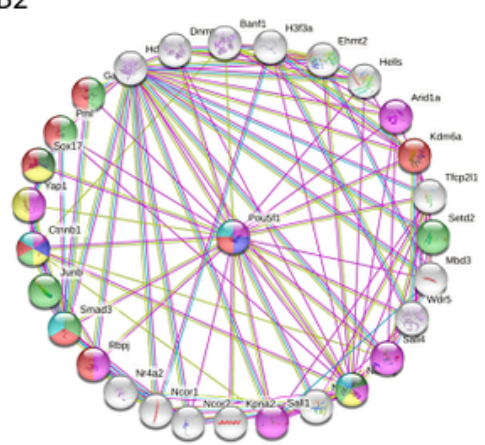

C1

\begin{tabular}{|c|c|c|c|c|}
\hline $\begin{array}{l}\text { GO-ferm and } \\
\text { pathways }\end{array}$ & Discerption & $\begin{array}{l}\text { Count in } \\
\text { network }\end{array}$ & FDR & Color \\
\hline 60:0007166 & $\begin{array}{l}\text { Cell surface receppor signaling } \\
\text { pattway }\end{array}$ & 8 of 174 & 0.0449 & \\
\hline GO:0051726 & Regulation of cell cycle & 7 of 101 & 0.0113 & \\
\hline GO:0019827 & Stem cell population maintenance & 8 of 138 & $3.01 \mathrm{e}-09$ & \\
\hline GO:2000144 & $\begin{array}{l}\text { Positive regulation of DNA- } \\
\text { templated transcription, intitiation }\end{array}$ & 2 of 38 & 0.0397 & \\
\hline GO:0072091 & Regulation of stem cell proliferation & 4 of 87 & 0.00045 & \\
\hline GO:2000035 & Regulation of stem cell division & 2 of 14 & 0.0083 & \\
\hline $\operatorname{mm} m 04550$ & $\begin{array}{l}\text { Signaling pathways regulating } \\
\text { pluripotency of stem cells }\end{array}$ & 9 of 137 & $3.97 e-12$ & \\
\hline
\end{tabular}

B2

\begin{tabular}{|c|c|c|c|}
\hline $\begin{array}{l}\text { Go--ternu and } \\
\text { pathways }\end{array}$ & Discerption & $\begin{array}{l}\text { Count in } \\
\text { network }\end{array}$ & FDR \\
\hline 60:0051302 & Regulation of cell division & & 0.0303 \\
\hline 60:003s198 & miRNA binding & 2 of 30 & 0.0341 \\
\hline G0:0005515 & Protein binding & 14 of 676 & 0.00077 \\
\hline 60:0007398 & Ectodem developancont & 20117 & 0.0069 \\
\hline 60:0048863 & Stem cell differentiation & 6 of 174 & 2.600 .06 \\
\hline GO:2000035 & Regulation of stem cell division & 2 of 14 & 0.0053 \\
\hline GO:0010646 & Regulation of cell communication & 10 of 321 & 0.0017 \\
\hline MMU-212436 & Generic Transcriptioa Pathway & 7 of 834 & 0.0084 \\
\hline
\end{tabular}

$\mathrm{C} 2$

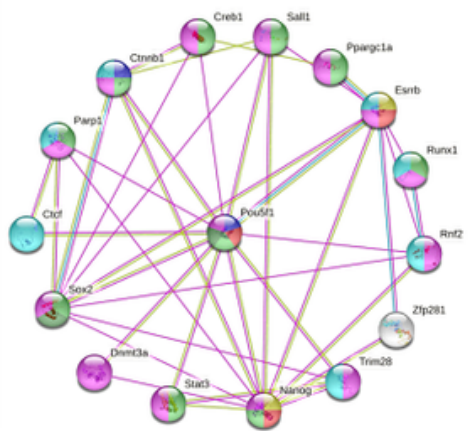

D1

D2

\begin{tabular}{|c|c|c|c|}
\hline $\begin{array}{l}\text { Go-term and } \\
\text { pathuays }\end{array}$ & Discerption & 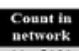 & $\mathrm{FDR}$ \\
\hline & $\begin{array}{l}\text { trive regultation of nucleic aci-i- } \\
\text { templated transcription }\end{array}$ & & $9.96-10$ \\
\hline 60:0006325 & Chromantin organization & 3 of 206 & 0.0086 \\
\hline GO:0045596 & $\begin{array}{l}\text { Negegtive regulation of cell } \\
\text { differentiation }\end{array}$ & 7 of 761 & $9,33 e-06$ \\
\hline 60:0010629 & $\begin{array}{l}\text { Negative regulation of gene } \\
\text { expression }\end{array}$ & 11 of 1811 & $1.77 e-08$ \\
\hline
\end{tabular}

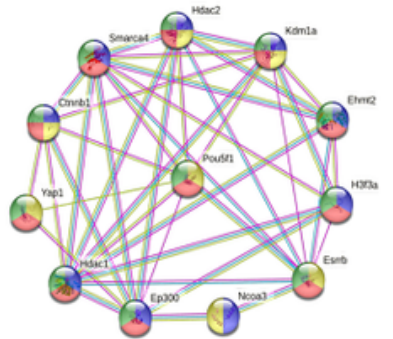

Figure 7

STRING Enrichment analysis. Each node represents a protein of our selected genes, and edges indicate protein-protein interactions, with line thickness indicating evidence strength for a predicted interaction. (a) 
biological enrichment of Pou5f1 in SSCs. (b) biological enrichment of Pou5f1 in ES-like. (c) biological enrichment of Pou5f1 in EBs. (d) biological enrichment of Pou5f1 in neurons.
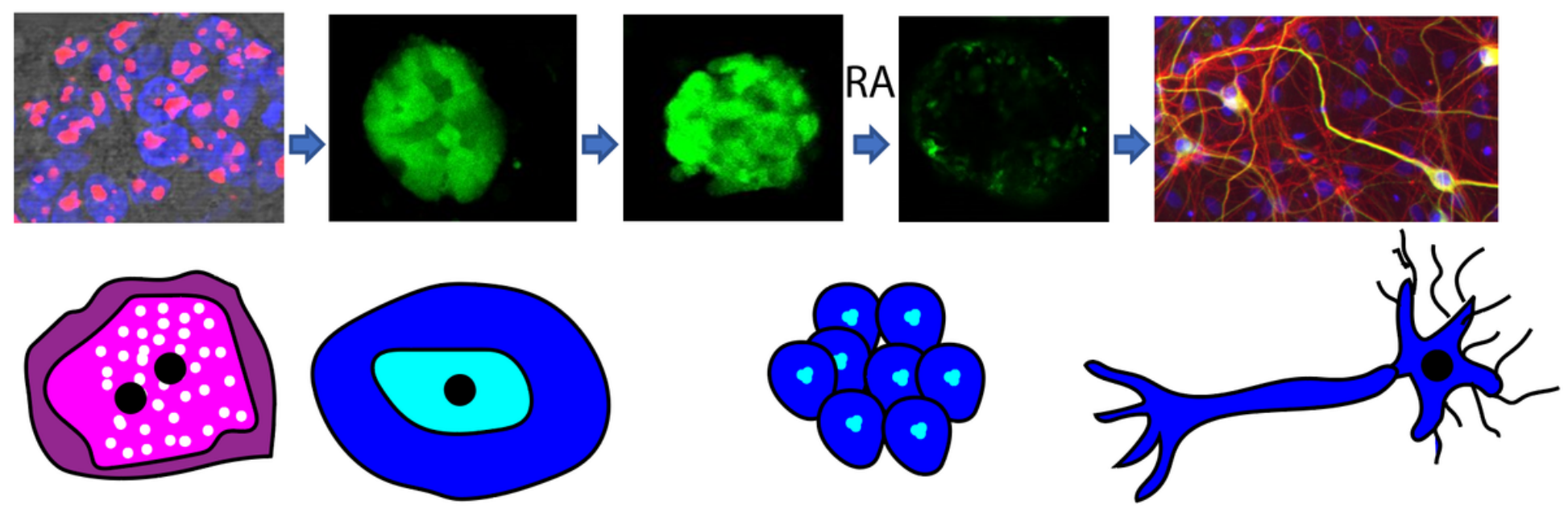

SSCS ES-likes

EBs

Neuron

\section{Oct-4}

Figure 8

Schematic of Oct4 Expression in the differentiation of SSCs into mature neurons process. Oct4 expression is high in SSCs, ES-like, and EBs but not expressed in neurons. 